\title{
Evaluation of General Dentists' and Dental Specialists' Knowledge about Oral Cancer in South Khorasan-Iran 2014
}

\author{
Narjes Akbari ${ }^{1}$, Vajehallah Raeesi ${ }^{2}$, Tahereh Khazaei ${ }^{3}$, Khaironnesa \\ Ramezanzadeh $^{4}$, Sediqe Ebrahimipour ${ }^{5 *}$
}

\begin{abstract}
Background: Oral cancer is one of the most prevalent cancers and one of the top ten causes of death in the whole world. Most oral cancers are diagnosed at late stages. Since dentists play a critical role in early detection of oral cancer, they should be knowledgeable and skillful in oral cancer diagnosis. The aim of this study was to survey dentist knowledge about oral cancer in Southern Khorasan Province. Materials and Methods: This descriptive, cross-sectional study was conducted with dentists who participated in an in-service educational program at the Faculty of Dentistry of Birjand University of Medical Sciences in spring 2014. A questionnaire including demographic information with 11 questions regarding oral cancer was prepared. The participants were required to be complete the questionnaires within a specific time span. The data were analyzed using SPSS 15 software by t-test and one-way ANOVA at 0.05 confidence level. Results: A total of 73 dentists out of 80 answered the questionnaires - $36(49.3 \%)$ were females and $37(50.7 \%)$ were males. Total mean score of knowledge was $7.91 \pm 1$ of 11 . Mean scores of knowledge of male and female participants were $7.70 \pm 1.83$ and $8.13 \pm 1.94$ respectively. Mean knowledge score of general dentists was $7.41 \pm 1.79$ and of dental specialists was $9.44 \pm 1.0$ In spite of higher knowledge score of women compared to men and general dentists compared to dental specialists, these differences were not statistically significant $(p=0.09)$. Tukey testing showed a significant difference between groups with 1-4 years of experience (8.74) and over twenty years of experience (6.50) $(\mathrm{p}=0.001)$. Conclusions: Considering the good knowledge level of young dentists and the specialists and the importance of early diagnosis of oral cancer, it seems necessary to pay more attention to academic education for dentistry students, as well as holding retraining courses for experienced dentists, so that their knowledge not be reduced over time.
\end{abstract}

Keywords: Dentist - knowledge - oral cancer - South Khorasan, Iran

Asian Pac J Cancer Prev, 16 (16), 6987-6990

\section{Introduction}

Oral cancer is one of the most prevalent cancers in human body; World Health Organization (WHO) has estimated that the incidence of oral cancer varies from 1 to 10 cases per 100,000 people in most of the countries (Shrivastava et al., 2014). Further, it has been realized that more than $50 \%$ of oral cancer patients access services in the advanced stages of disease (Warnakulasuriya, 2009) . Several etiologic factors are involved in its creation. Tobacco chewing, smoking and alcohol are the main reasons for the increasing incidence rates (Scully and Bagan, 2009; Halawany et al., 2013). Since oral cancer has variable clinical features in different stages, and may vary from an asymptomatic area to changes in the color and consistency, it often does not show clinical signs of malignancy and therefore is not recognized at early stages (Seoane et al., 2006). Early detection and treatment of malignant and dysplastic lesions is essential to making a good prognosis. Delay in diagnosing, makes it generally difficult or impossible to be treated (Onizawa et al., 2003). Unfortunately, despite the availability of oral cavity for examination and not requiring advanced tools and also not being uncomfortable for the patients, most cases of oral cancers are diagnosed when the symptoms appear because of the progress of the disease (Burket et al., 2008; Warnakulasuriya, 2009). This delay in diagnosis may result from disregarding and lack of adequate information of both patients and physicians in routine oral examinations.

Dentists are familiar with the structures and health of the oral cavity and are the first group who might examine patients for main signs and symptoms of oral cancer; so they can significantly enhance the life expectancy in patients suffering from oral cancers. Conventional oral examination still constitutes the gold standard screening

${ }^{I}$ Department of Oral Medicin, School of Dentistry and Dental Research Center, ${ }^{2}$ Department of Internal Medicine, ${ }^{3}$ Department of Anesthesia and Critical Care, Paramedical School, ${ }^{4}$ Education Research Center, ${ }^{5}$ Department of Endodontics, School of Dentistry and dental Research Center, Birjand University of Medical Scinces, Birjand, Iran., Iran *For correspondence: sdent22@bums.ac.ir 
tool for potentially malignant oral lesions and cancer. Interestingly, the findings of the most lasting (15-year) randomized controlled trial on oral cancer screening using visual examination supported the introduction of a screening program in high-risk individuals (Kujan et al., 2006)

Insufficient level of awareness is one of the important factors in the lack of accurate and timely diagnosis of oral cancer and potentially malignant lesions at the primary stages (Kujan et al., 2006).

Although plenty of surveys have been conducted to assess the dentists' awareness and behavior towards oral cancer (Yellowitz et al., 2000; Zarei and Asadpour, 2002; Alonge and Narendran, 2003; Motallebnejad and Hedayati, 2006; Saghafi et al., 2009; Sarabadani et al., 2012; Mehdizadeh et al., 2014), there is no conclusive study which comprehensively evaluates the dentists' awareness regarding oral cancers in south Khorasan. Thus the present study was designed to assess the dentists' and dental specialists' knowledge about oral cancer in south khorasan-Iran

\section{Materials and Methods}

In this descriptive, cross-sectional study all general dentists and dental specialists who were working in South Khorasan Province ( 80 person) and taking part in continuous education program in faculty of dentistry of Birjand University of medical sciences were participated.

Data were collected using a standard questionnaire in which the validity of the questionnaire was confirmed using content validity method ( 5 oral and maxillofacial expert evaluated the questionnaire) and test retest was performed to confirm the reliability $(\mathrm{r}=0.83)$

The questionnaire comprises two sections: 1) demographic data include age, gender, year of graduation, academic rank, university education and participating in retraining courses, 2) questions that assessed knowledge of diagnostic skills and predisposing factors of oral cancer (each correct answer were awarded 1 and wrong answer were given a zero score. The minimum acceptable score based on PUMA (Performance by unified model analysis) was considered as 7. This model used for evaluation of non-functional properties of a design (such as performance, dependability, security, etc.)

Therefore, awareness level of dentists who scored less than 7 would be undesirable and scores of 7 or higher would be desirable. From 80 distributed questionnaires 73 questionnaires were returned (response rate 91.25\%). Verbal consent was obtained from each participant. Collected data were analyzed using descriptive statistics (Mean, Standard Deviation, percent and Median) and Chi-square test, $t$ test and analysis of variance at 0.05 of confidence by SPSS 16 .

\section{Results}

A total of 73 dentists out of 80 , answered the questionnaire. Some of demographic features of participants are summarized in Table 1 of total participants, only 18 $(24.7 \%)$ attended in oral cancer retraining courses. Most

Table 1. Frequency of Demographic Characteristics of the Dentists

\begin{tabular}{llcc}
\hline Demographic & & Frequency & Relative frequency \\
\hline Gender & Female & 36 & 49.3 \\
& Male & 37 & 50.7 \\
Degree & General dentist & 55 & 75.3 \\
& Specialist & 18 & 24.7 \\
\hline
\end{tabular}

Table 2. The Mean of Knowledge Scores out of Total Score of 11 According to the Academic Degree and Experience

\begin{tabular}{lccl}
\hline $\begin{array}{l}\text { Characteristics } \\
\text { Academic Degree }\end{array}$ & Number & Mean \pm SD & Test \\
$\quad$ General dentists & 55 & $7.41 \pm 1.79$ & $\mathrm{t}=4.42$ \\
$\quad \begin{array}{l}\text { Dental specialists } \\
\text { Experience (year) }\end{array}$ & 18 & $9.44 \pm 1.29$ & $\mathrm{p}=0.09$ \\
$1-4$ & 31 & $8.74 \pm 1.67$ & $\mathrm{f}=5.18$ \\
$5-9$ & 11 & $7.27 \pm 2.00$ & \\
$10-14$ & 10 & $8.40 \pm 1.64$ & \\
$15-19$ & 5 & $7.80 \pm 2.38$ & $\mathrm{p} *=0.001$ \\
$>20$ & 16 & $6.50 \pm 1.31$ & \\
\hline
\end{tabular}

*The difference is statistically significant

Table 3. Answers of the Respondents to the Questions Regarding Knowledge about Oral Cancer

\begin{tabular}{|c|c|c|c|c|}
\hline \multirow[t]{2}{*}{ Question } & \multicolumn{2}{|c|}{ General practitionaire } & \multicolumn{2}{|c|}{ Dental specialist } \\
\hline & $\begin{array}{l}\text { Correct } \\
\mathrm{N}(\%)\end{array}$ & $\begin{array}{l}\text { Wrong } \\
\mathrm{N}(\%)\end{array}$ & $\begin{array}{l}\text { Correct } \\
\mathrm{N}(\%)\end{array}$ & $\begin{array}{l}\text { Wrong } \\
\mathrm{N}(\%)\end{array}$ \\
\hline 1 -SCC is the most common type of oral cancer. & $43(87.2)$ & $12(12.8)$ & $18(100)$ & $0(0)$ \\
\hline 2- Tongue is the most common site of oral cancer. & $39(70.9)$ & $16(29.1)$ & $15(88.9)$ & $3(16.7)$ \\
\hline 3- Smoking is the most important predisposing factor of oral cancer. & $51(92.7)$ & $4(7.3)$ & $16(88.9)$ & $2(11.1)$ \\
\hline 4- Submandibular lymph nodes are the first places of metastasis of oral cancer. & $49(89.1)$ & $6(10.9)$ & $18(100)$ & $0(0)$ \\
\hline 5- Lung is the most common site of distant metastasis of oral cancer. & $37(67.3)$ & $18(32.7)$ & $16(88.9)$ & $2(22.1)$ \\
\hline 6- Anterior hard palate is rarely affected by cancerous lesions. & $38(69.1)$ & $17(30.9)$ & $17(94.4)$ & $1(5.6)$ \\
\hline 7- Lower lip is the area with better prognosis of oral cancer. & 27(49.1) & $28(50.9)$ & $14(77.8)$ & $4(22.2)$ \\
\hline \multicolumn{4}{|c|}{ 8- floor of mouth is the most Likely area for malignant transformation of precancerous lesions. } & 2(11.1) \\
\hline \multicolumn{5}{|c|}{ 9- Lateral posterior portion of the hard palate Is the most common place for accurance of minor salivary gland tumors. } \\
\hline 10- The most common precancerous lesions in the oral cavity is leukoplakia. & $32(58.2)$ & $23(41.8)$ & $12(66.7)$ & $6(33.3)$ \\
\hline \multicolumn{5}{|c|}{ 11- 2 weeks is the minimum time needed to differentiate a malignant disease from an inflammatory lesion? } \\
\hline & $35(63.6)$ & $20(36.4)$ & $17(94.4)$ & $1(5.6)$ \\
\hline
\end{tabular}


of them $(87.7 \%)$, gained their knowledge about oral cancer during the academic study. Others mentioned retraining, self-study and the media. In spite of higher knowledge score of women compared to men and general dentists compared to dental specialists, the differences were not statistically significant $(\mathrm{p}=0.09)$. Scores of knowledge about oral cancer according to the academic degree and experience and answers to the questions are shown in table 2 and 3 respectively. $100 \%$ of dental specialists had favorite knowledge score. But in general practitioners $32.7 \%$ had undesirable and $67.3 \%$ had desirable knowledge score.

ANOVA test showed differences between groups with different work experiences and tukey test showed that this difference was significant between groups with 1-4 years experience (8.74) and over twenty years of experience (6.50) $(\mathrm{p}=0.001)$.

The knowledge score of the diagnostic skills of dentists was $2.8 \pm 0.84$ from 4 . The scores of females and males were $2.9 \pm 0.9$ and $2.8 \pm 0.76$ respectively. This difference was not statistically significant $(\mathrm{p}=0.3)$.

The mean knowledge scores of the diagnostic skills of dentists and dental specialists were $2.7 \pm 0.83$ and $3.4 \pm 0.61$ from 4 respectively. Although the specialists score was higher, this difference was not statistically significant $(p=0.3)$. The mean score of knowledge about the risk factors in females and males were $1.5 \pm 0.5$ and $1.4 \pm 0.5$ of 2 respectively. .

There were no statistically significant differences between the scores of diagnostic skills and work experience. $(\mathrm{p}=0.3)$

\section{Discussion}

In this study, we surveyed the dentists' knowledge about oral cancer in South Khorasan province for the first time.

The results of this study have shown that the overall level of knowledge of the dentists is favorite (7.91 \pm 1 of 11$)$

In spite of higher Knowledge score of females compared to males and general dentists compared to dental specialists, the differences were not statistically significant $(\mathrm{p}=0.09)$.

Colella et al. reported that performing an oral cancer screening is not usual procedure for dentists in Italy, with only $52.3 \%$ responding that such an examination would be conducted at the initial appointment for every patient (2008).

In this study, $87.2 \%$ of the general dentists and $100 \%$ of the specialists believed Squamous Cell Carcinoma (SCC) as the most common cancer of the oral cavity. The similar question was answered correctly by $78 \%$ of the dentists in the study of Motallebnejad et al. (2006) and 32\% in the study of Colella et al. (2008) and $81.2 \%$ of the dentists in the study of Mehdizade et al. (2014).

In the current study, $70.9 \%$ of the general dentists and $88.9 \%$ of the specialists selected the tongue as the most common site of oral cancer. Correct answer was pointed by $56.5 \%$ of respondents in the study of Motallebnejad et al (2006) and 50.5\% in the study of Colella et al. (2008) and $59.6 \%$ of respondents in the study of Mehdizade et al (2014). This difference is probably due to the lower average age of our study group.

The highest level of knowledge, was in the dentist with work experience of less than 5 years $(8.7 \pm 1.6)$, and the lowest level of knowledge was among dentists with over 20 years of experience $(6.5 \pm 1.3)$ and this difference was statistically significant $(\mathrm{p}<0.001)$. The Study by Ogden and mahboobi, also showed that the students of the final years showed greater knowledge of risk factors and clinical features of the disease (2011).

Considering that the majority of participants (7.87\%) in this study, have mentioned that they obtained their information during the educational period, plus that most dentists have fewer opportunities to study after graduation, these results are predictable. Since the majority of participant were young and a good knowledge score was obtained, it seems that training during the education is sufficient, and in retraining programs it is better to focus on the examination of soft tissues of the oral and lymph nodes of the head and neck region; so that, the diagnostic skills do not reduce over time.

Despite the higher score of specialists comparing to general dentists, this difference was not statistically significant. It is probably due to the small number of specialists participating in this study. Previously, there were no comparative studies between general dentists and specialists. It is suggested that studies are designed to evaluate the level of knowledge of specialists in a way that more participants are included.

In our study, $61(83.6 \%)$ of participants had referred patients with suspected lesions to oral medicine specialists or oral and maxillofacial surgeons. routine examination for oral cancer was conducted by $91.3 \%$ and $81 \%$ (Horowitz et al., 2000a; Horowitz et al., 2000b) Of general dentists in two studies carried out in the United States and by $92 \%$ of specialists in oral surgery, oral medicine, surgical dentistry and general dental practitioners in the UK (Kujan et al., 2006)

Superior awareness and oral cancer knowledge was identified in the community with access to the campaign materials (Croucher et al., 2011).

During the recent years with increasing numbers of dental experts and ease of access, it seems to be easier for dentists to refer their patients to relevant professionals. In this study, the level of knowledge for all expert participants was favorite and it is hoped that, with timely and proper referral of patients to specialists, lesions will be diagnosed at the early stages.

The prognosis of oral cancer is not satisfying. On the other hand, the oral cavity is readily available for examination and does not require advanced examination tools. Thus, it is expected that oral cancer be detected in its early stages by the dentists; but unfortunately most studies have shown that the disease is often diagnosed in advanced stages. lack of adequate knowledge is the main reasons for late diagnosis of oral cancer (Burket et al., 2008). Providing community based approach education programs and employing social policy are needed for preventing of cancer (Bayrami et al., 2013; Bayrami et al., 2014).

Due to the importance of early detection of 
Narjes Akbari et al

precancerous lesions and the important role of dentists, dental schools are expected to educate dental students to acquire an acceptable level of knowledge regarding oral cancer to help early diagnosis of the cases during future working years.

As the level of knowledge was higher in dentists with less years of experience who had been graduated more recently, the necessity of holding retraining courses for dentists is obvious. Besides, more emphasis should be placed on oral cancer prevention in dental schools.

\section{References}

Alonge O, Narendran S (2003). Opinions about oral cancer prevention and early detection among dentists practising along the Texas-Mexico border. Oral diseases, 9, 41-5.

Bayrami R, Taghipour A, Ebrahimipour H (2013). Challenges of providing cervical cancer prevention programs in iran: a qualitative study. Asian Pac J Cancer Prev, 15, 10071-7.

Bayrami R, Taghipour A, Ebrahimipour H (2014). Personal and socio-cultural barriers to cervical cancer screening in iran, patient and provider perceptions: a qualitative study. Asian Pac J Cancer Prev, 16, 3729-34.

Burket LW, Greenberg MS, Glick M, et al 2008. Burket's oral medicine, PMPH-USA.

Colella G, Gaeta GM, Moscariello A, et al (2008). Oral cancer and dentists: knowledge, attitudes, and practices in Italy. Oral Oncol, 44, 393-9.

Croucher R, Islam S, Nunn H (2011). Campaign awareness and oral cancer knowledge in UK resident adult Bangladeshi: a cross-sectional study. Br J Cancer, 105, 925-30.

Halawany HS, Jacob V, Abraham NB, et al (2013). Oral cancer awareness and perception of tobacco use cessation counseling among dental students in four Asian countries. Asian Pac J Cancer Prev, 14, 3619-23.

Horowitz A, Drury T, Canto M (2000a). Dental Practice: Practices of Maryland dentists: oral cancer prevention and early detection baseline data from 1995. Oral Diseases, 6 , 282-8.

Horowitz AM, Drury TF, Goodman HS, et al (2000b). Oral pharyngeal cancer prevention and early detection: dentists' opinions and practices. The Journal of the American Dental Association, 131, 453-62.

Kujan O, Duxbury A, Glenny A, et al (2006). Opinions and attitudes of the UK's GDPs and specialists in oral surgery, oral medicine and surgical dentistry on oral cancer screening. Oral Diseases, 12, 194-9.

Mehdizadeh M, Majidi MS, Sadeghi S, et al (2014). Evaluation of knowledge, attitude and practice of general dentists regarding oral cancer in sari, Iran. Iranian J Cancer Prev, 7, 101 .

Motallebnejad M, Hedayati M (2006). General dentist's knowledge about oral cancers in Babol, in 2005. J Mash Dent Sch, 30, 309-18.

Ogden GR, Mahboobi N (2011). Oral cancer awareness among undergraduate dental students in Iran. J Cancer Education, 26, 380-5.

Onizawa K, Nishihara K, Yamagata K, et al (2003). Factors associated with diagnostic delay of oral squamous cell carcinoma. Oral Oncol, 39, 781-8.

Saghafi S, ZareMahmoodabadi R, Salehinejad J, et al (2009). Evaluation of general dentists knowledge about oral cancer in Mashhad-Iran in 2008. J Mash Dent Sch, 33, 107-14.

Sarabadani J, Pakfetrat A, Delavarian Z, et al (2012). Knowledge and Diagnostic Skill of General Dentist in Khorasan Razavi Province Common (Iran) toward Oral Diseases 2009-10. J
Mashhad Dental School, 36.

Scully C, Bagan J (2009). Oral squamous cell carcinoma overview. Oral Oncol, 45, 301-8.

Seoane J, Warnakulasuriya S, Varela Centelles P, et al (2006). Oral cancer: experiences and diagnostic abilities elicited by dentists in North western Spain. Oral Diseases, 12, 487-92.

Shrivastava SR, Shrivastava PS, Ramasamy J (2014). Oral Cancer in developing countries: The time to act is upon us. Iranian J Cancer Prev, 7, 58.

Warnakulasuriya S (2009). Global epidemiology of oral and oropharyngeal cancer. Oral Oncol, 45, 309-16.

Yellowitz JA, Horowitz AM, Drury TF, et al (2000). Survey of us dentists'knowledge and opinions about oral pharyngeal cancer. J Am Dental Assoc, 131, 653-61.

Zarei M, Asadpour F (2002). Assessing dentist's knowledge and awareness in the diagnosis of oral cancer in Kerman. Shahid Beheshti Medical Sciences University JDental School, 4, 364-57. 